Voix et Images

\title{
En liberté, comme un papillon, un arbre! Entretien avec Paul-Marie Lapointe
}

\section{Michel van Schendel et Jean Fisette}

Volume 17, numéro 3 (51), printemps 1992

Paul-Marie Lapointe

URI : https://id.erudit.org/iderudit/200974ar

DOI : https://doi.org/10.7202/200974ar

Aller au sommaire du numéro

Éditeur(s)

Université du Québec à Montréal

ISSN

0318-9201 (imprimé)

1705-933X (numérique)

Découvrir la revue

\section{Citer ce document}

van Schendel, M. \& Fisette, J. (1992). En liberté, comme un papillon, un arbre! Entretien avec Paul-Marie Lapointe. Voix et Images, 17(3), 387-410.

https://doi.org/10.7202/200974ar d'utilisation que vous pouvez consulter en ligne.

https://apropos.erudit.org/fr/usagers/politique-dutilisation/ 


\title{
En liberté,
}

\section{comme un papillon, un arbre! Entretien avec Paul-Marie Lapointe}

\author{
Michel van Schendel et Jean Fisette* \\ Université du Québec à Montréal
}

Là où vient boire l'orignal.

JF - La nature est très présente dans votre poésie: les arbres et les animaux reviennent constamment dans Le Vierge incendié, puis dans les textes postérieurs. Et cet imaginaire, 'c'est le fait d'un type qui n'est pas originaire d'une ville, je pense. Le contact avec la nature a été important dans votre enfance?

PMI - Oui. Mais surtout avec la nature telle qu'elle a été dite et vécue par mes grands-parents maternels. Mon grand-père était menuisier; il possédait un atelier où se retrouvaient ses vieux copains. Il avait été l'un des fondateurs du village...

J'y allais très souvent; j'y ai passé une partie de ma tendre enfance. Ces gens-là racontaient des histoires fantastiques; la colonisation du Lac Saint-Jean, ça été quelque chose de phénoménal, une histoire remplie de personnages mythiques. Par exemple, on racontait qu'un bûcheron, un jour, avait reçu un arbre sur la tête; il s'était arc-bouté, avait enfoncé d'un pied ou deux dans le cran, c'est-à-dire dans le roc, puis s'était secoué et avait recommencè à travailler. Et encore, le Grand feu du Lac Saint-Jean; je me souviens, mon grand-père racontait que les gens plongeaient dans la rivière avec des roseaux dans la bouche pour pouvoir respirer au moment où le vent balayait l'incendie d'une rive à l'autre. Enfin, des histoires tenant de l'épopée. Et par dessus tout, il y avait ma grand-mère, avec laquelle j'allais à la pêche, presque tous les matins; je n'avais que deux ou trois ans, qu'elle m'amenait déjà pêcher le brochet sur la rivière Ashuapmuchuan...

- Avec la collaboration de Sandrine Donkers qui a procédé à la transcription de l'entretien. 
MvS - Ah! ça c'est un beau mot!

PML - Et qui signifie: «Là où vient boire l'orignal .

Par ailleurs, c'était une société très fermée. J'ai bien vite souhaité sortir du village. Non pas à cause de mes parents, au contraire, ce sont eux qui me retenaient, sentimentalement... mais à cause de l'atmosphère même du village, plutôt étouffante...

JF - Dans votre milieu familial, il y avait des affinités pour les arts? On dessinait, on écrivait, on faisait de la musique?

PML - Oui, du côté de mon grand-père paternel, qui était médecin. C'était une famille très, très particulière, vivant dans la musique... Surtout des filles (commé du côté de chez ma mère d'ailleurs)... J'ai appris la musique très jeune, le piano, pendant très longtemps. J'étais le premier petit-enfant de la famille; j’ai été chouchouté... Alors, je n'ai jamais oublié. Des femmes extraordinaires...

JF - Dans un monde de femmes à l'époque, le piano était toujours important.

PML - Il y avait aussi des violons, une mandoline, une clarinette, un accordéon...

JF - Alors, l'activité culturelle était présente!

PML - Oui. L'activité culturelle, je pense, la plus répandue au Québec à cette époque-là : la chanson, la musique. Une de mes tantes était organiste et mon père, maître de chapelle et clarinettiste; et puis il chantait la messe tous les matins. Ça été un drame de ma vie, obligé de servir la messe, très jeune, alors que mon père chantait dans le chœur; j'étais sous la surveillance de l'œil paternel... Mais c'était un homme très charmant, très doux...

JF - Et le jazz qui deviendra si présent dans l'imaginaire et l'écriture de votre poésie, il était présent à l'époque?

PML - Je me souviens... Il y avait dans le village, à la mi-carême, une fête, une mascarade sur la patinoire, pour laquelle les gens se costumaient. J'y avais gagné un prix, un album de disques 78 tours que j'ai conservé d'ailleurs - intitulé New 52nd Street Jazz. Ça été la découverte de Coleman Hawkins, de Dizzy Gillepsie, de quelques autres. C'était pendant la guerre; mes premiers disques de jazz, le début d'une passion qui dure...

MvS - Mais, tu devais déjà avoir une certaine connaissance, une certaine fréquentation du jazz. Enfin, il y avait la radio chez vous? 
PML - Oui. Mais il n'y avait pas beaucoup de jazz à la radio. La radio, c'était CKAC et CBJ la station de Chicoutimi de Radio-Canada. La radio a joué un rôle extraordinaire chez nous, il faut le dire. Elle aura été un instrument culturel fantastique. La radio, contrairement à la télévision qui vide, ça donne à rêver, à réfléchir, comme le livre...

JF - ... et à imaginer...

PML - ... à imaginer, c'est ça! Elle laisse place à l'imaginaire. Il y avait les émissions dramatiques, les radio-romans, Madeleine et Pierre, la musique, les chansons... Et puis, il y avait aussi les nouvelles de la guerre, du monde qui nous parvenaient. Je suis né en 1929. J'avais 11, $12,13,14$ ans pendant la guerre. Les informations venaient de Montréal et de Londres, on entendait le Big Ben, les correspondants de guerre... Alors on écoutait... Ça nous ramenait le monde...

JF - Cela devait être extrêmement impressionnant quand on était dans une région éloignée. Le monde rentrait dans la maison et donnait à imaginer?

MvS - Tu as raison d'insister sur le fait que la radio ne mange pas les gens. Elle suscite leur imagination et leur imaginaire et il y a peut-être une bonne raison à ça, c'est la voix radiophonique: la voix est la métaphore du corps, la voix fait rêver; la voix est métaphore du corps et en même temps, elle est soustraite du corps...

JF - Elle véhicule tout le corps.

MvS - C'est ça!

JF - Et elle laisse de la place...

PML - C'est le phénomène du langage en soi; et c'est exactement ce qui arrive avec le livre...

JF - ... et la parole de poésie. Je crois qu'elle se situe à quelque part entre la voix orale et le texte écrit. Je crois qu'on est dans cet espacèlà, n'est-ce pas?

PML - Oui!

JF - C'est en étudiant au séminaire de Chicoutimi que vous avez découvert la poésie; à ce moment-là, qu'est-ce que vous lisiez?

PML - On étudiait encore la littérature à l'époque, la littérature du passé: les Grecs, les Latins, les troubadours, Villon, Ronsard, Lamartine, Hugo, enfin tous. Et puis, il y avait l'Enfer, la bibliothèque interdite, où j'avais découvert les symbolistes, des anthologies, Proust... J'ai été un lecteur insatiable. 
MvS - Ça c'est au séminaire?

JF - Vous avez donc baigné dans la longue tradition de la poésie française?

PML - Oui.

JF - Et la poésie québécoise était présente?

PML - Parmi les poètes québécois, moi j'ai d'abord été impressionné - enfin, peut-être parce que je l'ai connu avant tout autre moderne par Saint-Denys Garneau. Je me souviens' avoir lu, au collège, Regards et Jeux dans l'espace, l'édition originale, et puis, à cause d'une photo d'elle, Anne Hébert, parce que je l'avais trouvée très belle...

JF - Oui. Mais Le Tombeau des rois, c'est un grand recueil...

PML - Mais à l'époque, j'ai été subjugué par sa beauté, ses yeux, plus que par sa poésie. Ensuite, j'ai été amené à mieux connaître Grandbois. Mais au moment où j'ai connu les premiers Québécois, c'est surtout Saint-Denys Garneau qui m'a frappé, parce que ça correspondait, à un moment tourmenté de ma vie, vers les 16,17 ans; chez Saint-Denys Garneau, je retrouvais la problématique de la société québécoise de l'époque. Tandis que Grandbois avait un peu échappé à ça.

JF - Est-ce qu'il y avait aussi un intérêt pour le livre, dans la vie familiale?

PML - Il n'y avait, pour ainsi dire, pas de livres à l'époque; ils étaient très rares, les livres. Très peu de livres parvenaient au Lac-Saint-Jean, sauf les dictionnaires, les manuels scolaires, les encyclopédies illustrées...

MvS - C'est exact.

PMI - C'est un phénomène...

MvS - ... qui est assez significatif.

PML - Par ailleurs, mon père, qui avait fait ses études classiques, était un peu l'écrivain public, chez nous, à Saint-Félicien.

MvS - Est-ce que c'était courant cette pratique dans les villages?

PML - Oui. Des gens avaient recours à quelqu'un qu'ils savaient instruit (on disait "qui avait une belle plume»), pour écrire, s'adresser aux administrations; aux institutions, répondre aux lettres...

MvS - Et ton père, c'était l'écrivain public?

PMI - C'est-à-dire que ce n'était pas officiel, mais les gens savaient qu'il pouvait écrire et il le faisait volontiers pour qui le demandait. 
JF - C'est très beau finalement. C'était l'intermédiaire entre les personnes non instruites et les institutions.

MvS - L'écrivain public n'était pas nécessairement un notable?

PML - Non, pas forcément. D'ailleurs mon père n'était pas un notable au sens où il y avait les avocats, les notaires, les médecins...

MvS - Mais il est devenu plus tard...

PML - ... il a toujours et, avant tout, été respecté.

Mvs - Comme gérant de...

PML - ... il était gérant de banque. Mais c'était un petit gérant d'une petite banque.

JF - Mais dans le village, c'était important.

PML - Oui, oui, c'était important.

La poésie est un travail de sape, il faut bien se le dire.

JF - Quand vous arrivez à Montréal, vous vous inscrivez au collège de Montréal, je crois?

PMI - Je suis venu au collège Saint-Laurent, dans la classe de philosophie. C'était en 1947. Puis, j'ai décidé d'aller à l'École des Beaux-Arts, parce que j'avais toujours été fasciné par la peinture; je m'imaginais que je pouvais devenir Van Gógh... Je suis entré aux Beaux-Arts et j'ai commencé à ce moment-là à écrire de façon plus sérieuse, ce qui allait devenir Le Vierge incendié.

JF - C'est là qu'on y arrive!

MvS - Ça date de quelle époquie?

PML — 1947, 1948. C'est à ce moment-là que j'ai décidé d'écrire...

MvS - ... tu as décidé d'écrire?

PMI - Oui.

MvS - Oui! Évidemment, c'est toujours une décision.

PMI - C'est ça, mais pas dans un but de publication. Je me suis aperçu que la peinture, ce n'était pas moi. L'écriture par ailleurs... 
MvS - Mais ce goût pour la peinture restait important?

PMI - Oui. La peinture a été une présence constante dans mon enfance; ma mère me racontait souvent que, devenue enceinte, elle avait eu une envie folle, une rage de peindre.

MvS - C'est pas vrai!

PML - Elle voulait devenir peintre. C'est très drôle; elle parlait de ça, comme d'un rêve. Une de ces folies, comme elle disait, qu'ont souvent les femmes enceintes...

Et elle l'a conservé tout le temps de sa vie, ce rêve. Elle répétait: "J'aurais donc voulu devenir peintre." Il faut rappeler qu'à l'époque, il y avait des peintres ambulants, dans les campagnes, qui faisaient des tableaux sur place et les vendaient aux gens; ainsi, il y avait trois tableaux de la région chez nous, des paysages de forêts, de chutes, qui avaient été achetés par ma mère. Par amour de la peinture, justement. Donc, je pense que la peinture, ça été déterminant pour moi, dès l'enfance, et ça l'est resté encore aujourd'hui, en particulier la peinture de ma femme, Gisèle, qui a été à l'origine de Tableaux de l'amoureuse.

MvS - ... ça vient de loin!

PMI - ... ça vient de très loin.

MvS - Ça vient de ton enfance.

PML - Tout vient de notre enfance...

JF - À l'époque où vous avez fréquenté l'École des Beaux-Arts, estce que vous étiez en contact avec Borduas et son groupe?

PML - Non! C'est l'École des Beaux-Arts que je fréquentais... Puis, pour des raisons familiales et économiques, j'ai décidé de ne pas me diriger vers la peinture, mais plutôt vers l'architecture. C'était l'année suivante, en 1948. J'ai rencontré là Robert Blair et Jean Lefebvre qui sont devenus des copains... Robert Blair, 'qui avait lu mes textes, m'a dit: "On va montrer ça à Gauvreau " (il avait étudié avec Claude ąu collège Sainte-Marie). C'est comme ça que j'ai-rencontré les automatistes. Gauvreau a aimé mes textes. Puis, il a été décidé de publier Le Vierge incendié.

MvS - Oui. Gauvreau, lui, déjà à ce moment-là, avait commencé d'écrire. Ses premiers objets dramatiques, tu te rappelles, datent de 1946... C'est-à-dire avant même la pleine gestation du mouvement automatiste et du manifeste le Refus global.

PMI - Gauvreau, c'était l'Écrivain du groupe. 
JF - Une question, qui m'intrigue depuis longtemps, monsieur Lapointe. Dans une entrevue donnée il y a longtemps vous aviez raconté qu'après votre rencontre avec Gauvreau, le texte du Vierge incendié avait été complètement réécrit en quelques jours...

PMI - C'était un tas de feuillets dans un grand cahier... Quand il a été question de publication, j'ai tout repris. Je n'ai pas réécrit, mais réaménagé; c'est-à-dire que, en quelques jours, j'ai refait l'ordre, j'ai réparti les poèmes en chapitres, j'en ai fait un livre quoi.

JF - La question est celle-ci: cette reprise, pour préparer l'édition, c'est vous seul qui l'avez faite? Gauvreau n'a rien eu à faire dans ce travail?

PML - Non, non, il n'a rien eu à faire dans ça! Non! il a simplement dit: ‘C'est formidable, il faut publier!• À ce moment-là, j'ai retravaillé mon manuscrit en vue d'une publication.

MvS - Est-ce que tu pourrais parler aussi de l'expérience de cette publication?

PML - Ça a été extraordinaire!

MvS - Je sais que les automatistes formaient vraiment un groupe, qu'ils bâtissaient une très forte solidarité. Et tout le monde mettait la main à la pâte... Pour la publication du Vierge incendié et également pour les autres publications, le Refus et Projections libérantes de Borduas.

PMI - Oui, en fait, Claude a été, si on peut dire, le premier à accueillir le texte et à suggérer l'idée d'un livre. Ensuite, il n'a plus rien fait au niveau de la publication. C'est Pierre Gauvreau qui, avec moi, a travaillé là-dessus et surtout Maurice Perron. Maurice Perron, qui était avec le mouvement automatiste: un photographe exceptionnel. Maurice a même avancé l'argent; il était le seul à gagner des sous parmi nous. Pour ma part, je vivais avec les deux ou trois dollars par semaine que mes parents m'envoyaient. Alors, Maurice avait fourni 100 \$ pour acheter le papier et louer la . Gestetner *. À l'époque, je vivais dans une chambre, rue Amherst, près du parc Lafontaine. J'y avais installé la *Gestetner * et on imprimait le soir, après les cours, Pierre Gauvreau, Maurice Perron et moi. Évidemment, au cours de cette période, j'ai été amené à rencontrer un peu plus souvent les autres automatistes.

MvS - Et les peintres du groupe, par exemple Mousseau, est-ce qu'ils mettaient la main à la pâte, aussi? Parce que lui m'a beaucoup parlé de ces expériences-là? 
PMI - Mousseau est même venu habiter, rue Amherst. Il vivait à l'époque avec Dyne. Tout le monde mettait la main à la pâte. Oui, c'était formidable!

JF - C'est tout à fait de cette façon-là que Refus global a été produit aussi.

PML - Je n'étais pas là moi, pour cette production.

JF - Vous n'aviez pas encore contacté le groupe, lorsqu'ils ont produit Refus global?

PMI - Non. En fait, je dois dire que toutes les activités des automatistes, ça m'impressionnait, mais ça ne m'intéressait pas tellement. J'étais plutôt pris par ma propre histoire; et puis je n'avais pas connu la même évolution...

MvS - C'est ce dont j'ai toujours eu l'intuition...

PML - Ils étaient beaucoup plus politiques, plus avancés que moi sur le plan de la connaissance. C'était presque tous des gens assez instruits: Claude Gauvreau, entre autres, avait une culture considérable. Puis, il y avait pire: ils étaient athées et, pour eux, c'était naturel. Tous, ils étaient très urbains...

MvS - Et toi, tu l'étais pas'?

PMI - J'étais encore pris dans mon passé villageois... je n'étais pas pratiquant... je vivais une sorte de crise, j'avais 18 ans...

MvS - Et l'un des moyens et des objets de la crise, c'était l'écriture du Vierge incendié?

PML - Bien oui, c'était une façon de m'en sortir...

MvS - Mais comment expliques-tu que d'une part, tu n'étais pas vraiment suscité, si je peux dire, par le mouvement automatiste qui était déjà assez fortement constitué et que, d'autre part, même si l'écriture du Vierge incendié était, m'a-t-il semblé, très autonome, par rapport à l'écriture de Claude Gauvreau par exemple, il y avait tout de même une forme d'accointance. Est-ce que tu peux parler de cette accointance?

PML - ... une accointance qui venait d'un besoin d'ouverture sur l'extérieur. Aux Beaux-Arts, avant de rencontrer les automatistes, j'avais fait la connaissance de Jean Benoît, Mimi Parent; Jean Benoît, mon premier professeur de dessin, m'avait prêté des livres, dont Capitale de la douleur (Éluard), et je suis entré ainsi en contact avec les surréalistes; c'est peut-être par là qu'on se rejoignait. J'avais lu Éluard puis Reverdy. 


\section{MvS - Et René Crevel?}

PMI - Non, pas à l'époque, je savais qu'il existait, mais je n'avais rien lu de lui. Reverdy a été plus important pour moi. Évidemment, il ne faisait pas partie du mouvement surréaliste, mais il y avait quelque chose d'assez extraordinaire dans le contact avec le monde réel chez Reverdy.

MvS - Et Breton? Tu étais moins attiré par Breton? Moins que Claude Gauvreau, en fait?

PML - Moi, les théoriciens m'intéressaient moins. Pour moi, chez Breton, il y avait d'abord le théoricien. Gauvreau, c'était un écrivain, un dramaturge, mais aussi un théoricien. Moi, pas du tout...

JF - Est-ce que Gauvreau vous avait communiqué les textes qu'il avait écrits, Les Entrailles?

PMI - J'ai vu des choses de lui, parce 'qu'on se rencontrait à l'occasion... Mais on n'échangeait pas de textes...

MvS - Est-ce qu'il était déjà un peu pape?

PML - Il en a toujours eu l'allure mais...

MvS - ... enfin, disons que c'est la réputation qu'on lui a faite plus tard, surtout à l'époque de l'exposition La Matière chante.

PML - Il aimait beaucoup Breton.

MvS - Oui!

PML - Peut-être, au fond, était-il le Breton d'ici.

MvS - C'est là, je pense qu'il ỳ a un petit détail qui est assez significatif, qui permettrait peut-être de toucher du doigt, l'une des différences d'orientation entre l'écriture du jeune Lapointe et celle du jeune Gauvreau. Dans cette écriture-là, celle du jeune Gauvreau, il y avait une orientation assez théoricienne. Effectivement, elle n'apparaît pas dans Le Vierge incendié, sauf que, tout de même, elle est implicite par la forme même de la discontinuité.

PMI - Peut-être... En tous cas, Gauvreau était très articulé, très savant... Il avait lu Freud, il avait lu les philosophes, il avait tout lu. C'était un garçon très cultivé.

JF - Une dernière question sur Le Vierge incendié: je pense que la richesse de ce texte tient, entre autres, à cet aspect dionysiaque dans la représentation de la sexualité et de la violence, à cette image carnavalesque qui est donnée de la société. Tous ces événements, autour de la publication du Vierge incendié, puis autour des manifestations 
des automatistes, tout cela se passe en bas de la ville, dans ce qu'on appelait alors le quartier du "Red Light *. C'était très différent de ce que pouvait être le village de Saint-Félicien...

PML - C'est ça - Montréal, la grande ville, ce fut un choc terrible, pour moi - mais, ce fut, en même temps, la remise en question de toute une orientation, de toute une civilisation... Tu sais, quand on parlait de la "grande noirceur ", c'était vrai. Pour sortir de là, il fallait quelque chose d'énorme.

MvS - Et la chose énorme, c'était l'arrivée déchirante à Montréal?

PML - Oui et la découverte de la possibilité de tout remettre en question, par l'écriture ou par quelque autre activité créatrice de même nature. En fait, le sens de "poésie", je pense que c'est là que je l'ai trouvé. Il faut que la poésie soit subversive, qu'elle se pose contre l'ordre établi; qu'elle remette en question le monde dans lequel tu vis, son langage...

MvS - C'est en quoi, fondamentalement - et très profondément la poésie est une affirmation. Mais elle affirme contre, et simultanément, elle affirme pour, pour elle-même...

PML - En somme, il y a là une prise de position essentielle contre la parodie de la vie... Il y a la vie, la petite vie, tu ne peux pas y échapper, malheureusement, sinon par une autre vie, la Vraie Vie, celle-là !

MvS - Exactement !

JF - La poésie est une affirmation forte.

PMI - Oui, c'est une recherche de...

MvS - ... ce n'est pas une recherche, c'est une découverte...

PMI - ... c'est une découverte de la vraie vie ou c'est une tentative d'exprimer ce que pourrait être la vraie vie...

MvS - Tu connaissais Rimbaud?

PMI - Évidemment. Rimbaud a été déterminant!

MvS - Ça été déterminant. Ah, bon. Tu peux parler de ça un moment?

PMI - Rimbaud, pour moi, ça été l'«illumination " de l'âme, la révélation de l'écriture salvatrice. J'ai trouvé chez Rimbaud le pur Adolescent, ouvert, intransigeant, révolté, la volonté absolue d'être...

La poésie c'est la voix de l'individu dans la foule, le cri de la solitude de l'être dans la société. La poésie, c'est un discours anti-social. La poésie est anti-socialé, quoique tu veuilles... 
MvS - Mais pas * asociale *

PML - Disons 'qu'elle est anti-société plutôt qu'anti-sociale. Chez Rimbaud, il y a ce phénomène. Au-delà de l'écriture, la poésie ouvre de nouvelles avenues à l'individu placé dans une société donnée; la poésie c'est la révolte, la subversion; elle est contre l'asservissement aux règles artificielles de la société. La poésie te permet de retrouver ton être profond...

MvS - J'aimerais tout de même essayer de te faire préciser un tout petit peu plus. Tu découvres Rimbaud et c'est le choc, comme ça été le cas pour les surréalistès français et belges, mais pas pour les Tchèques, je ne crois pas. Puis il y a Éluard, celui de Capitale de la douleur et, plus tard, celui du Livre ouvert; il y a là, je dirais, une pensée très sensible du social. Et pourtant, c'est une pensée libérante. Tu disais que la poésie est "anti-société," beaucoup plus qu'«antisociale....

PMI - Enfin, Éluard, c'est le phénomène de la tendresse et de l'amour; il n'y a pas pour moi de contradiction entre le fait d'être « antisociété", de remettre en question la société et celui d'aimer les êtres humains; ce ne sont pas les êtres qui sont en cause, mais l'organisation des collectivités, c'est le poids des collectivités, c'est le poids des organisations sociales.

MvS - Voilà !... et des appareils...

PML - ... je pense que cette dimension humaine, on la trouve chez Éluard, et qu'elle explique son évolution erratique $\longrightarrow$ on peut le dire maintenant. Sa poésie vient d'une grande générosité qui s'alimente à la tendresse, à l'amour des hommes...

MvS - ... qui est vécu, par Éluard, non pas simplement par l'écriture mais aussi dans sa vie. C'était un être d'une immense générosité.

JF - Pour que la poésie soit significative; il faut qu'elle puisse être échangée et, poúr qu'elle puisse être échangée entre les personnes, il faut qu'elle soit fondamentalement sociale, mais elle s'affirme contre les organisations formelles de la société.

PML - Oui! Vous abordez là, je crois, le phénomène de la communication... on entre dans le domaine de la qualité et de la quantité... On peut être social, sans atteindre le plus grand nombre... Je pense que, par le travail de création, on peut ne toucher qu'une infime partie des gens. Il ne faut pas s'inquiéter des répercussions nombreuses ou peu nombreuses des poèmes. La poésie est un travail de sape, il faut bien se le dire. C'est en cela que la poésie, comme n'importe quelle œuvre 
d'art véritable, est subversive; à partir du moment où elle est intégrée, elle n'est plus efficace en regard de la créativité et du progrès qu'elle peut apporter.

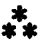

Tu découvres, en essayant de vivre comme tout le monde, que c'était bien vrai, que ton intuition était bien juste. Et, à ce moment-là, il faut revenir à l'écriture, aller encore plus loin dans la subversion.

JF - Monsieur Lapointe, en préparant cette entrevue, j'ai pris conscience du fait suivant: il y a des blocs dans votre vie, des périodes de création séparées par des plages assez longues de silence. D'abord les œuvres de jeunesse en 1948-1949 avec Le Vierge incendié et Nuit [...]; puis une période de silence; puis, au début des années soixante, Arbres et Pour les âmes et un autre silence qui nous mène jusqu'en 1973 où vous nous donnez une série de textes qui culminent avec les écRiturEs en 1980; et depuis un nouveau silence. Il y a trois périodes d'écriture puis de longues périodes de silence. On retrouve le même Paul-Marie Lapointe dans les différentes époques avec des variantes... Mais' ces plages de silence, comment les expliquer? Vous n'avez pas écrit? Vous n'avez pas publié? Ou bien vous étiez trop occupé ailleurs?

PML - Il faut dire que je suis bien paresseux, très paresseux, c'est une de mes caractéristiques et j'y tiens beaucoup. Mais, surtout, il y a les exigences du quotidien, du terrible quotidien. Gagner son pain au jour le jour. Il s'agit de la malédiction première pour l'homme, qu'il doive se vendre pour survivre. Bon! telle a été mon histoire avant tout, ce qui explique les pauses, le rythme de la production: j'ai gagné ma vie, expression mensongère s'il en est, mais tirée telle quelle du discours social.

JF - Mais le métier de journaliste que vous avez commencé de pratiquer en revenant à Montréal au début des années soixante... Étiez-vous à l'aise dans ce métier-là?

PML - Je suis devenu journaliste à Québec en 1950; c'était la seule avenue qui s'ouvrait à moi. Par ailleurs, ce métier 'm'a passionné; je crois y avoir été utile. Et il m'aura permis de prendre contact avec le monde réel, de renforcer par une meilleure connaissance de la vie des 
hommes, ici et ailleurs, ma conviction de la nécessité absolue de la poésie. On ne peut être subversif que si on aime les gens. Et pour aimer les gens, il faut savoir à quoi ils sont soumis... Tu ne peux pas faire de poésie si tu n'es pas impressionné par le monde, par l'univers... Même la poésie de Mallarmé n'est pas abstraite. C'est par rapport à des circonstances très concrètes que Mallarmé a écrit; ce n'était pas indépendant de sa vie quotidienne, qui était terrible aussi, d'une certaine façon.

Alors ces pauses de poésie, c'est le gagne-pain, mais aussi des temps de réflexion, de mise en quarantaine un peu forcée sans doute...

MvS - Et, en même temps, c'est un phénomène de gestation, parce que c'est aussi productif...

PMI - En effet. C'est très productif et puis moi, je ne me considère pas comme un écrivain écrivant automatiquement. Mon occupation dans la vie n'aura pas été que ça... Je n'y aurai pas "gagné ma vie "...

MvS - Gagner ton sel! Je préfère l'expression "gagner son sel. à "gagner sa vie „. "Gagner sa vie”, c'est horrible.

JF - C'est avec la poésie que l'on gagne sa vie.

PML - Oui, c'est ça. Mais je tenais à utiliser l'expression *gagner sa vie "; n'est-ce pas ce qu'on dit, quand on te demande de trouver un boulot, alors que ce serait plutôt la "perdre" en définitive... mais ça, c'est une autre histoire...

MvS - Je reviens sur la question de l'intensité. Je prends l'exemple de Nuit [...J. Tu as écrit ça très, très rapidement... Et pourtant, c'est un texte extrêmement organisé. Mais tu l'as écrit très rapidement comme si'ça venait d'un seul jet. Et pourtant, c'était pas vraiment de l'automatisme. Il y a là, une écriture extrêmement intense, au moins aussi intense que celle du Vierge incendié. Puis cette expérience d'écriture conduit à un silence. Comment expliquer cela ?

PMI - Nuit [...], ce fut un cul-de-sac! Et un aboutissement; j'avais atteint la limite de mes possibilités d'écrire; il me fallait chercher ailleurs, me consacrer à autre chose. Devenu journaliste, je m'y suis consacré entièrement.

MvS - Ça aussi, c'est intéressant!

PMI - Ces pauses marquent aussi des évolutions. Il n'y a pas de rupture, même s'il y a des pauses. En fait, on continue à cheminer, mais autrement. Et puis, vous le remarquiez tout à l'heure, on retrouve à peu près les mêmes rythmes de silence et de création entre Le Vierge incendié et écRiturEs. Il y a une continuité. 
JF - Il y a, à l'évidence, des correspondances.

PML - Sans doute, parce qu'on retrouve dans les deux recueils le même fond de subversion. Mais tu es aussi exacerbé par les pauses. Tu découvres, en essayant de vivre comme tout le monde, qué c'était bien vrai, que ton intuition était bien juste. Et, à ce moment-là, il faut revenir à l'écriture, aller encore plus loin dans la subversion, ne plus se contenter d'un discours de la subversion, mais aller jusqu'à la subversion du discours...

[...] la poésie, c'est une célébration, une fête, un jeu; c'est de la subversion... c'est une improvisation de jazz, aussi...

Mvs' - On trouve pourtant des points d'ancrage à ton métier de journaliste. J'allais très souvent chez toi à l'époque - c'est le moment où on s'est connu -, tu me montrais un long rouleau de papier journal sur lequel tu avais écrit Arbres. C'était du papier qui provenait des téléscripteurs, un papier vert.

PML - Des bouts de rouleau vert; ça ne pouvait plus servir. Moi, je récupérais ces bouts de rouleau, d'autant plus qu'il y avait un papier carbone, donc une copie en même temps...

MvS - Il y a un rapport au métier même et à la matérialité du métier de journaliste que tu pratiquais à ce moment-là.

PML - Oui, tu as raison. D'ailleurs, je n'écris qu'à la machine à écrire, je ne fais pas de manuscrit. Mes seuls manuscrits, ça été Le Vierge incendié, parce que je n'avais pas de machine à écrire à l'époque. Je l'ai ensuite repris à la machine à écrire en vue de l'impression. Et puis, il y a le rythme de la machine à écrire, qui est important dans ma poésie.

MvS - C'est très important, parce qu'il y a un autre ancrage: l'activité, elle aussi très intense et très systématique, de l'auditeur, de l'écouteur de jazz. Et il me semble que, tout comme le rythme de la pratique sur la machine à écrire, l'écriture s'accompagne nécessairement de pauses, de pauses qui sont marquées, qui sont sonores et qui rejoignent, en tout cas dans ton écriture, les syncopes du jazz. 
PMI - C'est intéressant ce que tu dis. Aussi la machine à écrire a un rôle inhérent, essentiel à ma poésie! Je ne m'étais jamais arrêté à cette dimension-là. Oui, c'est vrai, tout ça se tient au fond, oui.

Journaliste - on tapait à trois doigts à l'époque -, je n'avais pas appris la méthode, je ne possédais pas, comme au piano, le doigté. Et pourtant, à la machine à écrire, j'arrivais à taper vite; et puis oui, il y a un rythme là-dedans, oui... et ça s'inscrit peut-être aussi dans la discontinuité. Au fond les pauses, c'est la dialectique dont on parlait tout à l'heure, entre la vie quotidienne et la remise en question de la vie quotidienne. Mais il y a aussi une continuité dans l'exercice du métier que tu fais. C'est pas inutile tout ça, il n'y a rien d'inutile.

JF - Car, de toute façon, il ne peut.y avoir de rupture totale entre la vie quotidienne et l'écriture de la poésie.

PML - Absolument pas!

JF - La suite Arbres, c'est un papier sans rupture?

PML - Oui!

JF - Cela a été fait sur une feuille sans rupture!

MvS - Sans rupture!

PMI - D'un seul coup d'ailleurs.

JF - La suite Arbres, c'est une célébration du mot, c'est-à-dire que le texte est continu mais... en même temps, ce sont des mots et expressions qui sont mis en évidence, des dénominations qui viennent d'une encyclopédie de botanique en grande partie. Il y a un côté encyclopédique dans ce texte-là. C'est la célébration du mot, par rapport à la phrase, par rapport à l'organisation du discours. Le poème est constitué d'une seule phrase, mais tellement longue que finalement on n'en perçoit que des fragments...

PML - Oui. C'est une célébration, c'est vrai. C'est très juste de dire ça. De toute façon la poésie, c'est une célébration, une fête, un jeu; c'est de la subversion, mais c'est aussi autre chose, et de beaucoup plus vaste. C'est une improvisation de jazz, aussi...

MvS - Exactement!

PML - Moi, je pense que la suite Arbres, c'est un poème qui vient directement du jazz. C'est du Coltrane...

JF - Et c'est un texte qui a été directement écrit à la machine à écrire. C'est-à-dire que c'est une improvisation des doigts sautant sur des touches? 
PMI - Oui, tu te donnes des thèmes qui t'impressionnent, qui suscitent en toi la fête, la célébration. Mais la technique n'est pas consciente; elle est, disons, naturelle, et c'est là qu'il faut parler d'artisanat préalable et d'influence: le jazz, l'improvisation du jazz.

JF - Et c'est la vitesse de la machine à écrire qui commande le rythme de l'écriture, il y a là quelque chose à mettre en relation avec l'automatisme de l'écriture...

PMI - ... et la vitesse de la machine à écrire, le rythme de la machine à écrire, c'est ça !

JF - La machine à écrire, puis le rythme dans le jazz qui prévalent sur l'imagination harmonique.

MvS - Oui, mais je voudrais peut-être dire ceci. C'est important ce que tu dis Paul-Marie par rapport au jazz et qui passe dans cette écriture de la suite Arbres. C'est pourquoi tout à l'heure, je disais beaucoup plus que l'auditeur de jazz, c'est l'écouteur de jazz. L'écoute c'est actif, c'est un principe d'activité d'écoute que tu mets en acte par l'écriture.

PMI - C'est très intéressant! Je pense qu'en fait, tous les arts s'influencent. Qu'est-ce qui fait chacun des arts? Tu as la technique et tout l'artisanat préalable, d'accord; mais il y a surtout le phénomène même de la création, qui est commun aux créateurs de tous les arts...

MvS - ... c'est l'intertextualité fondamentale.

PML - Bien, oui.

MvS - De l'art.

PMI - Il faut s'alimenter partout. Si tu n'es pas inspiré par le quotidien directement, il faut puiser ton inspiration ailleurs. D'où les pauses dont on parlait plus tôt: il faut pouvoir s'abstraire d'un certain lieu pour pouvoir mieux en parler. Il faut que tu partes de quelque chose de positif, il faut que tu té retrouves, il faut que tu aies des racines aussi, de la parenté quelque part. Alors, moi je retrouve tout ceci dans les arts, la musique, la peinture...

JF - Et cette écoute dont vous parlez, c'est la même dont on parlait plus tôt à propos de la radio? L'écoute qui ouvre l'imaginaire, qui est, en quelque sorte, très créatrice...

PMI. - L'aspect de l'écoute est fondamental. La perception du monde, ça passe par les sens, il faut bien se le dire: la visualité, l'ouïe, la tactilité... En somme, c'est une tentative de retrouver ce qui est ton corps...

MvS - Tu as parlé du discours comme d'un moment nécessaire, enfin comme d'un point d'aboutissement. C'est en même temps une 
relance, qui suit et précède une gestation; elle aussi est extrêmement active, car elle participe constamment d'une écoute active. Il y a dans Arbres, un phénomène discursif extrêmement intéressant. C'est que cet immense texte est composé, si on le prend dans le détail, d'une phrase non terminée, une phrase substantivée où l'emporte le nom et parfois l'adjectif. L'invention, le déferlement d'images toutes discontinues, mais toutes cohérentes, entre elles; et pourtant cette longue suite est ordonnée comme une longue phrase. Et il y a d'ailleurs des moments de temps forts dans ce texte; parmi les moments forts, il y a la récurrence constante de "j'écris arbre*. "J'écris arbre*, c'est l'amorce, c'est le programme même de cette longue phrase.

PMI - C'est intéressant comme analyse. C'est un discours qui est, disons, «jazzé». Ce n'est pas le discours dit cohérent, le discours rationnel, c'est un discours qui vient du corps du monde, c'est un discours concret; c'est pour ça que "J'écris arbre " ne parle pas d'autre chose que de lui-même; c'est un discours qui n'appartient pas au langage quotidien: un discours-jazz, un discours musical, un discours qui prend ailleurs sa dynamique.

JF - Est-ce qu'il n'y a pas là quelque chose de la litanie?

PMI - Oui, c'est ça, c'est de la litanie! Oui, de la mềme façon que l'improvisation de jazz est litanique, tu reviens sur le même thème, en le transformant peu à peu...

MvS - ... ou en procédant par variations.

JF - La discussion que nous avions tenue, Michel et moi, et qui nous a conduit à la notion d'*oraison " repose sur ces considérations: on trouve Pour les âmes, "Psaume", puis "Janvier - Quête de Chaleurs" qui commence ainsi: "Chaleur nous te prions". En somme, on trouve tout un vocabulaire emprunté au discours religieux. J'avais envisagé le terme "prière", mais Michel a suggéré, avec raison je pense, le terme "oraison "...

MvS - ... parce que ce terme a beaucoup plus de gravité, il renvoie plus au sacré qu'au religieux. Dans *oraison ", il n'y a aucune soumission; puis on y retrouve l'aspect de la discursivité.

JF - Je compare les trois périodes d'écriture: d'abord, Le Vierge incendié et $N u i t$ [...J; puis les textes des années soixante-dix; et, en position intermédiaire, les textes des années soixante qui sont les plus cohérents, les plus discursifs. Est-ce que j'aurais raison de dire qu'à cette époque de la Révolution tranquille, au début des années soixante, c'est le moment où Paul-Marie Lapointe est le plus en accord - le moins en révolte, disons - avec la discussion qui a lieu sur la place publique? 
PMI - La Révolution tranquille marquait certainement une ouverture pour nous. Et on ne peut nier que l'artiste, le poète fonctionne aussi à partir des ouvertures qui se manifestent sur le plan social, parce que ces ouvertures s'inscrivent dans le sens de la liberté, de la pleine réalisation de l'homme...

JF - Et à cette époque, il y avait un partage de l'écriture avec certains discours reconnus sur la place publique: le discours contestataire, le discours de renouvellement, le discours critique porté sur notre société.

PML - Je suis fondamentalement d'accord pour que l'homme d'ici puisse se réaliser dans une société qui lui convienne, mais je n'ai pas de recette qui soit autre que poétique. Il faut être absolument ouvert à tout. Mais, qu'est-ce qui va faire qu'on vive enfin dans une société d'ouverture, d'amour, de compassion? J'ai horreur de la société contemporaine, de sa violence essentielle, du peu de place qu'elle fait à l'âme de chacun de ses membres. Contre la violence de cette sociétélà, il faut être d'une intolérance absolue, jusqu'à la subversion.

MvS - Tu parles très justement de l'ouverture. L'ouverture, c'est une dialectique et il y a toute sorte de formes, c'est d'une incroyable fécondité, cette dialectique de l'ouverture. Par exemple, Jean, tu faisais référence, tout à l'heure, au discours religieux. Â l'époque où j'ai connu Paul-Marie, donc en 1956, il était chroniqueur religieux à $L a$ Presse, et il l'est resté jusqu'à son départ en 1958. Il est donc resté chroniqueur religieux durant plusieurs années. Et c'est à cette époque aussi que les textes de la suite Arbres et de Choix de poèmes sont en gestation. Et plus tard, dans Pour les âmes, on retrouve le phénomène de l'oraison. Et l'oraison, c'est précisément une dialectique, elle est ancrée dans l'exercice du métier et, en même temps, elle s'inscrit contre le discours religieux.

PMI - Oui, je pense! Je n'ai d'ailleurs rien inventé. Depuis les débuts de l'humanité c'est un peu comme ça que ça se passe... Le genre litanique, tu le retrouves dans toutes les littératures primitives...

MvS - ... * dites " primitives.

PMI - "dites ", oui, oui! Parce que là - il faut bien le préciser -, quand on parle de primitivisme, on ne parle pas de quelque état d'infériorité, mais de pureté, d'intégrité, d'une absence de cette corruption qui nous afflige.

MvS - Ce serait plus juste de dire que c'est du discours paralogique. C'est-à-dire qui procède d'une autre logique. 
PML - Quand on parle de la poésie, on entre dans le domaine du sacré, où il n'y a pas de raison raisonnante... La parole est pour personne, elle est pour tout, pour l'ambiance... C'est un message donné qui ne s'adresse ni à Dieu, ni aux hommes...

MvS - Ni à Dieu, ni au diable!

JF - C'est l'interprétant.

MvS - C'est l'interprétant de Peirce.

JF - Ce n'est pas un destinataire, c'est l'interprétant.

PMI - Dans toute œuvre véritable de création, tu ne te mets pas aux pieds de celui à qui tu t'adresses...

JF - Nous ne sommes pas dans une logique de communication, nous sommes dans une logique de signification. Ça été la première phrase dans mon cours l'automne dernier: "La poésie ne communique pas, elle signifie."

PML - C'est exact!

MvS - La poésie est un acte.

PML - Le grand drame de la communication aujourd'hui, c'est que ça ne signifie plus.

JF - Voilà, c'est ce que je crois aussi.

PML - À ce propos, l'activité journalistique est éloquente: le contenu du message n'est déterminé souvent que par le nombre de lecteurs qu'il génère. La qualité de la communication n'est plus mesurée qu'à l'aune de l'efficacité marchande.

JF - Alors on ferme les potentialités de signification, en se situant uniquement dans une logique de communication.

PML - C'est ça! L'écriture, en définitive, sert à ouvrir des possibilités de signification. Parce qu'il faut trouver de nouvelles significations.

[...] la poésie porte en soi sa propre inefficacité, sa propre inutilité. Mais, en même temps, c'est un acte essentiel.

JF - Nous arrivons au troisième bloc, aux textes qui vont des Tableaux de l'amoureuse en 1973 jusqu'aux écRiturEs en 1980. 
En relisant le texte des Fragments/Illustrations, j'ai eu l'impression d'une certaine amertume par rapport à l'usure, à la banalisation de la parole publique. Vous vous livrez à une caricature acerbe des discours médiatiques de l'information, de la politique et de la publicité. À l'époque du Vierge incendié, cet usage de la parole figée, sclérosée, marquée idéologiquement, fermée, répétitive existait à sa façon et alors la poésie, détournant la signification, marquait une révolte. Dans écRiturEs et les Fragments/Illustrations, la critique est plus fondamentale, car il semble que ce soit la possibilité même d'écrire qui soit remise en cause... Est-ce qu'il y a une amertume?

PML - Non! Je ne pense pas qu'il y ait de l'amertume dans mon cheminement; je suis passé à l'extrême remise en question du discours idéologique. Il s'agit d'une inscription absolue dans ce que je crois être la subversion, la révolte contre ce discours-là. Évidemment, ça pose un certain nombre de problèmes. Par ailleurs, cette position rejoint toute une série de mouvements historiques qui ont été déterminants, comme le dadaïsme.

JF - Dans cette série de texte, la ligne est très nette, la déconstruction est de plus en plus marquée et puis, lorsqu'on arrive à écRiturEs, la subversion est totale. À l'époque du Vierge Incendié, on trouvait une puissance, une force. Mais à l'aboutissement de cette série qui est celui d'écRiturEs, et c'est appuyé par le texte des Fragments/Illustrations, on a l'impression que c'est la possibilité même d'écrire qui est remise en cause.

PMI - Ce n'est pas la possibilité d'écrire qui est remise en cause, mais la nature même d'une écriture qui serait la répétition plus ou moins habile d'un message déjà connu, banalisé. Il y a là une logique: la contestation s'inscrit dans la nature même de l'écriture.

MvS - Par rapport à tout ce dont nous parlons, on pourrait lire un passage des Fragments/Illustrations. On y trouve, précisément enchassée, la parole marchande, la parole mercantile. Je le lis, si vous me permettez:

Impuissants? Unanimes? Cent millions d'êtres sont assis devant les messages multicolores tonitrués: rince-bouches pour le baiser, fausses semelles contre les miasmes, gouvernement-du-peuple-par-le-peuplepour-le-peuple, merveilleux savons, femmes-robots, bières enchantées à rengaines-scies, fromages doucereux à joie de vivre, antisudorifiques et gomme fraîche, dentifrice automobiles prêts-au-consommateur voyagesdans-les-îles, 33-étoiles-du-football-pendant-des-heures, pirates de l'air bonbons chocolats, Idi Amin croustilles dodo. Vietnam et demain matin goulags petit déjeuner, Palestiniens droits de l'homme liberté d'entreprise grand premier ministre, nourriture-à-chiens-à-chats meurtre crapuleux 
carrière dans la vente. Intérêt public. Chansons, discours, bestsellers.

Marchandises. La vie. Toute la vie.

PMI - C'est un poème ça?

MvS - Bien sûr que c'est un poème!

JF - Oui, c'est l'usure totale, la banalisation...

PML - C'est ça, contre la fausseté du discours quotidien.

JF - Et qu'est-ce qui reste comme possibilité à la poésie après cela?

PML - Justement, il faut chercher des avenues. Moi, ces avenues, je les vois dans la signification, c'est-à-dire que, par la poésie, il faut trouver de nouvelles façons d'être. J'ai dit ailleurs que ça n'exclut pas la dimension politique. Évidemment, nous ne parlons pas de la classe des hommes politiques... parce que c'est dégueulasse, ce qui se passe partout, il n'y a pas un pays au monde qui échappe à cette engeancelà. C'est la fausseté du discours en soi. Mais il est sûr, qu'éventuellement, il faudra une Politique, il faudra que le monde existe à partir des notions d'amour, de tendresse, d'amitié... Qu'il soit organisé à partir de ces notions-là... Mais est-ce qu'on y atteindra jamais?

JF - Finalement, la question est celle-ci : comment trouver le moyen, avec l'écriture, de ne pas sombrer dans le discours dominant qui est celui de la caricature, et qui puisse le renouveler?

PMI - Ça dépend des talents et de la volonté de chacun. Il y a là une contradiction fondamentale. Ce qui nous ramène à la dialectique. La poésie est une contestation nécessaire et constante du monde; mais elle doit partir du monde, de la vie, venir au monde; or la poésie ne pourra jamais être un discours populaire et cohérent; elle porte en soi sa propre inefficacité, sa propre inutilité. Mais, en même temps, c'est un acte essentiel, car si la poésie ne peut pas servir directement à l'organisation de la société, elle doit servir à la transformation des individus, donc éventuellement, de la société.

JF - En lisant le texte des Fragments/Illustrations, on en arrive quasiment à cette image de deux mondes parallèles: le monde réel et le monde de l'imaginaire, comme s'il n'y avait plus de pont entre les deux mondes. Et puis, on regarde le texte d'écRiturEs et on a aussi cette impression-là. écRiturEs a laissé tout le monde pantois, interrogatif et moi aussi, comme les autres. Est-ce qu'il n'y a pas une rupture totale entre le monde de l'imaginaire, ludique certes, mais qui risque de devenir assez formel et d'autre part, ce monde du réel qui est le comble de la banalisation... 
PMI - Il y a une rupture, forcément, en toute bonne logique. Mais ça s'inscrit dans la temporalité ça aussi. Et, il y a l'avenir. Plus la société est invivable, plus s'impose la quête d'une autre réalité, qui doit venir de l'imaginaire. Parce que les recettes appliquées pour améliorer le sort de l'humanité - de tous les hommes - ne se sont pas révélées très efficaces jusqu'à présent. Il faut chercher ailleurs. Non. pas dans l'irréel, mais dans une autre approche du monde.

Que le texte soit difficile, déconcertant et même incompréhensible, cela va de soi. Mais n'est-ce pas aussi son objet et sa raison d'être qu'il ne puisse être avalé, digéré par la société qu'il conteste?

JF - Puis vous arrivez aux mots croisés: le mot croisé, c'est l'énigme?

\section{PMI - Oui!}

JF - C'est là la signification des écRiturEs?

PML - C'est cela aussi. À toutes les époques de l'histoire de l'humanité, vous retrouvez l'énigme.

JF - Oui!

PML - Il n'y a pas une civilisation qui n'ait eu sa dimension énigmatique, ses mystères. C'est essentiel. Il n'y a pas de civilisation qui vive sans mystère. Sinon vous tombez dans la banalisation. Évidemment, nous autres, Occidentaux du $\mathrm{XX}^{\mathrm{e}}$ siècle, nous sommes des rationalistes. Nous acceptons mal ces réalités-là. Mais moi, je ne vois pas pourquoi le sacré serait toujours identifié à l'aspect exploiteur des religions, à une naïveté de primitifs... Le mystère fait partie de la vie. Il grandit même à mesure que la science se développe.

JF - Mais ce mystère que je vois dans l'écriture, il nous est donné de façon extrêmement abrupte, sans contenu en fait?

PMI - Bien oui, il y a un contenu. C'est plein de choses là-dedans, de "sens", de nouvelles réalités qui n'ont pas de références dans le quotidien, mais qui surgissent d'une nouvelle façon de nommer le monde, littéralement. Le scandale, c'est que les mots ont un poids de réalité dont ils ont été vidés, une réalité qui se perd dans le langage usuel; ils ne signifient plus au delà du cliché.

Je me souviens d'une étudiante qui m'avait téléphoné de Québec... ça fait au moins 10, 15 ans; dans son cours, on lui avait demandé de commenter un texte du Réel absolu. Imaginez, ce n'était même pas écRiturEs. - "Le soleil est bleu ". Qu'est-ce que ça veut dire? — Ça veut dire exactement ce qui est écrit : "Le soleil est bleu * - Mais, ça n'existe pas un soleil bleu. - C'est écrit, donc ça existe dans ce texte: 
"Le soleil est bleu". Ainsi, il faut lire littéralement, oublier les références au discours habituel, prendre le contenu de chacun des mots pour lui-même, et s'ouvrir aux réalités nouvelles ainsi créées...

JF - Au lieu de simplement renvoyer, le langage qui crée d'une façon absolue...

PMI - Oui, c'est ça. Le langage est rempli de possibilités, d'ouvertures pour l'avenir même du quotidien... Sinon à quoi ça servirait toutes ces œuvres à travers les âges, qui n'ont aucune signification immédiatement connue.

JF - En définitive, écRiturEs n'a fait que pousser à bout la logique qui était celle du Vierge incendié en 1948?

PML - D'une autre façon, oui! Au moment du Vierge incendié, il y avait quand même un désir d'intégration à une société ou de transformation d'une société. Je ne veux pas dire qu'à mon âge, ce désir n'existe plus. Au contraire, il reste toujours présent. Mais je ne le vivrai pas, sans doute, cet univers transformé. Même s'il y a des indices, vous les signaliez tout à l'heure: la curiosité des gens, le besoin d'en savoir plus sur ce qui n'est pas le quotidien, et puis l'organisationnel, peut-être...

JF - Le besoin d'un déplacement de la sensibilité et de l'imaginaire. Alors, la poésie doit se protéger contre les tentatives de récupération, elle représente la révolte absolue.

PML - Pour moi, c'est ça, parce que je crois avant tout à la liberté. La liberté, c'est un absolu. Il y a tellement de limitations dans notre vie que, $s^{\prime}$ il y a un domaine où il faut ne pas en avoir, c'est bien dans celui de la création...

JF - Pour vous, la poésie est d'abord, et de façon dominante, une parole, une oralité? Je reviens sur l'oraison, je reviens sur le jazz, je reviens sur la rythmique de l'écriture, je reviens sur l'aspect non figuratif de l'écriture qui est une potentialité. Tout cela, ce ñ'est pas l'usure...

PML - Mais là, vous parlez de la forme plutôt que de ce qu'est la poésie en soi. La poésie, ça génère du sens, ça va donc au-delà... \& Le soleil est bleu ", c'est aussi un sens, c'est une ouverture sur le sens. L'aspect formel, c'est peut-être le phénomène de l'oralité, mais qui nous mène à autre chose. Et pour moi, l'autre chose est très importante, c'est la liberté, l'ouverture des possibles. Comment pourrait-on résumer ça? As-tu une formule, Michel?

MvS - Non, non, pas du tout. 
PML - Moi, je dirai que la poésie, c'est une fête. Il faudrait que la vie soit une fête. Il faut que ça soit amusant de vivre sur la terre, que ça soit un plaisir. Il y a l'oralité dans ça, il y a la notion de plaisir écrit, parlé, pensé, imaginé... La fête quoi!

JF - Vous allez vous remettre à écrire après avoir pris quelque mois de repos?

PML - Oui, peut-être, je ne le sais pas...

MvS - Loin, très loin dans la nature...

PMI - À mon âge, on a envie de prendre du recul. Je voudrais simplement m'arrêter pour penser...

JF - Vous retirer, c'est ça?

PMI - Je ne vais pas me retirer du monde, mais vivre en retrait, si on peut dire...

Mvs - Oui!

PMI - Voilà comment je veux être. Dans la Nature! Un être humain en liberté... comme un papillon, un arbre! 